\title{
Efecto del contenido de azufre y del grado de desoxidación sobre la ductilidad en caliente de aceros inoxidables austeníticos resulfurados en estado de solidificación
}

\author{
J. Botella ${ }^{(*)}$ y R. Sánchez ${ }^{(*)}$
}

\begin{abstract}
Resumen La fabricación de aceros inoxidables austeníticos de alta maquinabilidad presenta una problemática específica derivada de su elevado contenido de azufre; elemento necesario para generar en la matriz austenítica inclusiones que faciliten las diferentes operaciones de mecanizado, pero perjudicial en cuanto al deterioro que produce en la deformabilidad en caliente. Este artículo describe el estudio realizado para evaluar el efecto del contenido de azufre y el grado de desoxidación sobre la ductilidad en caliente de aceros inoxidables austeníticos resulfurados, partiendo de una estructura de solidificación (as cast condition). Se realizaron ensayos de tracción en un sistema Gleeble, a temperaturas entre 1.150 y $1.250^{\circ} \mathrm{C}$, analizándose el parámetro de ductilidad en función del contenido de azufre, nivel de desoxidación, y del tipo, tamaño y distribución de los sulfuros presentes. Los resultados ponen de manifiesto el efecto perjudicial de los contenidos de azufre y oxígeno sobre la deformabilidad en caliente de los aceros inoxidables austeníticos resulfurados, y la necesidad de controlar especialmente el nivel de óxidos de dichos aceros.
\end{abstract}

Palabras clave: Acero inoxidable austenítico resulfurado. Alta maquinabilidad. Deformabilidad. Desoxidación.

\section{Effect of both sulphur content and deoxidation degree on the hot ductility of resulphurized austenitic stainless steels in the solidified state}

\begin{abstract}
The manufacture of free machining austenitic stainless steels features a specific drawback derived from their high sulphur content, which is needed for generating, into the austenitic matrix, inclusions to optimize the different machining operations. However, sulphur has a harmful effect on hot workability. This paper deals with assessing the effect of sulphur content and deoxidation level on the hot ductility of resulphurized austenitic stainless steels in as cast condition. Hot tensile tests were conducted on a Gleeble machine, at temperatures between 1,150 and $1,250{ }^{\circ} \mathrm{C}$, studying a ductility factor as a function of sulphur content, deoxidation degree, as well as type, size and distribution of sulfides. Results point out the harmful effect of increasing sulphur and oxigen contents on the hot workability of resulphurized austenitic stainless steels, and the need to control carefully the level of oxides of these steels.
\end{abstract}

Keywords: Resulphurized austenitic stainless steels. Free machining. Workability. Deoxidation.

\section{INTRODUCCIÓN}

El procedimiento más utilizado para optimizar la capacidad de mecanización (por torneado, fresado, etc.) de los aceros es la adición de determinados contenidos de azufre y manganeso, de forma que se

\footnotetext{
* Centro I+D de Acerinox, S.A. Fábrica del Campo de Gibraltar, Aptdo. 83. 11370-Los Barrios (Cádiz, España).
}

asegure la formación de inclusiones tipo $\mathrm{MnS}$, en masa proporcional a la del azufre de aleación. Estos sulfuros, distribuidos uniformemente en la matriz del acero, constituyen una fase que actúa a modo de lubricante para la pieza de corte, permitiendo tanto una mayor vida útil de ésta, como una superior velocidad de mecanizado, en relación al acero sin resulfurar. 
El grado de resulfuración de los aceros es variable, en función del objetivo concreto de mejora de la maquinabilidad, y presenta, en cualquier caso, un determinado compromiso entre dicho objetivo y el resto de propiedades del material. En el caso de los aceros inoxidables, la presencia de sulfuros de manganeso afecta a la corrosión por picaduras y el azufre es, por sí mismo, un elemento residual que reduce considerablemente la capacidad de conformado del acero a alta temperatura.

De cara a la deformabilidad en caliente del acero inoxidable austenítico resulfurado, el azufre tiene un doble efecto. Por un lado, el azufre en solución solida (azufre libre) se encuentra segregado en los bordes de grano y en los bordes $\delta / \gamma$, y puede dar lugar, en función de su contenido, a la descohesión de dichos bordes (1). Este efecto se debe a que el tamaño del átomo de azufre es relativamente grande (2) y su solubilidad en la matriz austenítica es aproximadamente del $0,01 \%$ a temperatura ambiente (3). En este sentido, la ductilidad en caliente de aceros inoxidables con ya muy bajos contenidos de azufre (10 a $20 \mathrm{ppm}$ ) se puede mejorar si se alcanzan niveles por debajo de $3 \mathrm{ppm}$ (2).

En segundo término, los sulfuros de manganeso tienen una determinada capacidad de deformación en relación con la matriz $\gamma$, y este hecho influye también en la deformabilidad global de la aleación. Habitualmente, los sulfuros de manganeso son suficientemente plásticos para acompañar la deformación de la austenita, pero pueden verse afectados por la presencia de otras inclusiones no metálicas asociadas a aquellos, que en los aceros inoxidables son normalmente óxidos. Estos reducen la deformabilidad de las inclusiones mixtas óxido-sulfuro, que llegan a constituir lugares preferentes de nucleación de grietas (4). De hecho, la utilización de tierras raras (TR) en la fabricación de aceros resulfurados ha estado principalmente justificada por la mejora de ductilidad en caliente. Las TR consiguen tal efecto más por el aumento de desoxidación que producen que por su efecto en la composición o morfología de los sulfuros (4).

En este estudio se han considerado las variables contenido de azufre e índice de limpieza de inclusiones tipo óxidos para determinar la deformabilidad en caliente de aceros inoxidables austeníticos resulfurados.

\section{MATERIALES}

Los materiales en estudio son muestras procedentes de palanquillas de colada contínua, y sus composiciones químicas se ofrecen en la tabla I. El estudio comparativo se realiza, por una parte, sobre cuatro materiales tipo AISI-303 $(C, D, E$ y $F)$ con niveles variables de oxígeno, entre 77 y $181 \mathrm{ppm}$. Por otra, el clásico AISI-304 $(A)$ es un austenítico de referencia, y junto con el AISI-316-L con 0,035 $\% \mathrm{~S} \cdot(B)$ y el 303 identificado como $C$, permiten el estudio del efecto del contenido gradual de azufre para niveles de desoxidación prácticamente constantes. En los materiales tipo 303, las variaciones composicionales (salvo el oxígeno en estudio) son discretas y no ejercen efectos cuantificables sobre la ductilidad en caliente a las temperaturas estudiadas.

\section{PROCEDIMIENTO EXPERIMENTAL}

El estudio realizado sobre las muestras de aceros austeníticos con distinto grado de resulfuración consta de dos partes principales. La primera, relativa a la caracterización del material de recepción (as cast), y la segunda, a los ensayos de ductilidad en

TABla I.- Composición química de las aleaciones en estudio (\% en peso)

TABLE I.- Chemical composition of the alloys under study (weight \%)

\begin{tabular}{|l|c|c|c|c|c|c|}
\hline & A (AISI-304) & B (AISI-316L) & C (AISI-303) & D (AISI-303) & E (AISI-303) & F (AISI-303) \\
\hline $\mathrm{C}$ & 0,022 & 0,040 & 0,059 & 0,054 & 0,067 & 0,060 \\
$\mathrm{Si}$ & 0,29 & 0,41 & 0,62 & 0,39 & 0,33 & 0,43 \\
$\mathrm{Mn}$ & 1,51 & 1,59 & 1,84 & 1,90 & 1,76 & 1,89 \\
$\mathrm{P}$ & 0,029 & 0,035 & 0,039 & 0,030 & 0,032 & 0,030 \\
$\mathrm{~S}$ & 0,001 & 0,035 & 0,294 & 0,308 & 0,304 & 0,317 \\
$\mathrm{Cr}$ & 18,35 & 16,66 & 17,35 & 17,12 & 17,67 & 17,07 \\
$\mathrm{Ni}$ & 8,74 & 11,24 & 8,63 & 8,75 & 8,16 & 8,52 \\
$\mathrm{Mo}$ & 0,28 & 2,12 & 0,39 & 0,43 & 0,22 & 0,30 \\
$\mathrm{~N}$ & 0,0481 & 0,0481 & 0,0522 & 0,0325 & 0,0482 & 0,0777 \\
$\mathrm{Cu}$ & 0,50 & 0,59 & 0,41 & 0,22 & 0,66 & 0,72 \\
$\mathrm{O}$ & 0,0070 & 0,0068 & 0,0077 & 0,0118 & 0,0134 & 0,0181 \\
\hline
\end{tabular}


caliente y al posterior análisis de resultados. En la fase de caracterización, se determinaron los contenidos de ferrita delta (mediante ferritoscopio) y de inclusiones no metálicas, la distribución de sulfuros en la sección transversal de las palanquillas $B$ a $F, y$ la composición (por microanálisis EDX) de inclusiones representativas.

Previo al mecanizado de las probetas para los ensayos de ductilidad en caliente, se procedió a reproducir sobre barretas de sección $15 \times 15 \mathrm{~mm}$ (y eje longitudinal paralelo al eje de la palanquilla), un tratamiento de precalentamiento equivalente al que reciben las palanquillas de austenítico en el horno de laminación. Se consideraron tres posibles temperaturas máximas en dicho horno, $1.220,1.250 \mathrm{y}$ $1.280{ }^{\circ} \mathrm{C}$, con salida del mismo a $1.190,1.220 \mathrm{y}$ $1.250{ }^{\circ} \mathrm{C}$, respectivamente. Dos probetas $(10 \mathrm{~mm} \varnothing$ $\times 120 \mathrm{~mm}$ ) por cada material y temperatura de salida fueron ensayadas a tracción en el sistema Gleeble, evaluándose la ductilidad en función de la reducción de área transversal tras la rotura, \% R.A.

\section{RESULTADOS}

En relación a la distribución de sulfuros en los materiales de medio $(B)$ y alto $(C$ a $F)$ nivel de resulfuración, las impresiones Baumann revelaron en todos los casos distribuciones finas y regularmente repartidas en la sección transversal.

La medición magnética de ferrita delta se realizó igualmente en la sección transversal de cada muestra de palanquilla. Los valores promedio de 64 medidas por muestra se indican en la tabla II. Tras el tratamiento de homogeneización, se comprobó que la ferrita había sido eliminada por completo en todos los materiales, por lo que los ensayos de ductilidad se realizaron posteriormente sobre estructuras totalmente austeníticas.

En la tabla II aparecen también los resultados del recuento metalográfico de inclusiones no metálicas. Las inclusiones tipo sulfuro no han sido con-

TABLA II.- Valores de ferrita delta medidos magnéticamente (en \% vol.), y de ocupación superficial de inclusiones no metálicas del tipo óxido (\% en área, según ASTM E 562-89) en los materiales en condición de solidificación

TABLE II.- Delta ferrite content measured by magnetic device (volume \%), and surface fraction of type oxide, non metallic inclusions (in area percent, to ASTM E 562-89 standard)

\begin{tabular}{|l|l|l|c|c|c|c|}
\hline & \multicolumn{1}{c}{$\mathrm{A}$} & \multicolumn{1}{c}{$\mathrm{B}$} & $\mathrm{C}$ & $\mathrm{D}$ & $\mathrm{E}$ & \multicolumn{1}{c|}{$\mathrm{F}$} \\
\hline $\mathrm{Fe}-\boldsymbol{\delta}$ & 8,9 & 2,4 & 1,8 & 1,4 & 1,4 & 0 \\
\hline Inclusiones & 0,025 & 0,046 & 0,029 & 0,054 & 0,066 & 0,102 \\
\hline
\end{tabular}

sideradas en los aceros resulfurados $(B$ a $F)$. Se aprecia una correspondencia significativa entre el nivel de desoxidación y el contenido de inclusiones tipo óxido. Las inclusiones presentes en la muestra $A$ son óxidos complejos. En los materiales $B$ y $C$, las inclusiones tipo óxido están presentes en cantidades similares a las del $A$, pero casi siempre asociadas a sulfuros, normalmente en su interior. En las muestras $D, E$ y $F$ se han observado también mayoritariamente inclusiones mixtas óxido-sulfuro. En relación con su tipología y distribución, los sulfuros de las muestras $B, E$ y $F$ corresponden al tipo I, uniformemente repartido, como es habitual en los materiales resulfurados. En la muestra $C$ se han observado principalmente sulfuros del tipo II, de distribución interdendrítica. En la muestra $D$, si bien la mayoría son tipo I, también se han encontrado sulfuros tipo II. Estos resultados son coherentes con los niveles de azufre y oxígeno de las aleaciones.

En la tabla III se indican los resultados de ductilidad de los ensayos de tracción en caliente realizados a $1.190,1.220$ y $1.250{ }^{\circ} \mathrm{C}$, y $5 \mathrm{~s}^{-1}$, en el sistema Gleeble. Los valores de \% R.A. indican, como era de esperar, un mejor comportamiento del austenítico tipo $304(A)$. Entre 6 y 7 puntos porcentuales por debajo, excepto a $1.190{ }^{\circ} \mathrm{C}$, se sitúa el AISI-316-L. En el grupo de los 303, se puede establecer una diferencia clara entre el resultado del material $C$ y los del resto. En el primer caso, y aun siendo en general menores que en los 304 y 316-L, se consigue un nivel de ductilidad considerablemente alto. Por el contrario, los valores de ductilidad son, con significativa diferencia, inferiores en las aleaciones $D, E$ y $F$, en los que no se sobrepasan resultados superiores al $70 \%$ de R.A. La figura 1 muestra gráficamente los resultados de \% R.A. en función de la temperatura.

\section{DISCUSIÓN DE LOS RESULTADOS Y CONCLUSIONES}

Para relacionar convenientemente los resultados de ductilidad y niveles de inclusiones de los materiales en estudio, se analizan en primer lugar el

TABLA III.- Valores de ductilidad (\% R.A) obtenidos a las distintas temperaturas de ensayo

TABLE III.- Ductility values (reduction-in-area percent) at the test temperatures

\begin{tabular}{|c|c|c|c|c|c|c|}
\hline & A & B & C & D & E & F \\
\hline $1190^{\circ} \mathrm{C}$ & 88,7 & 87,9 & 77,7 & 64,3 & 62,1 & 53,6 \\
$1220^{\circ} \mathrm{C}$ & 94,7 & 87,3 & 78,5 & 67,2 & 66,9 & 55,4 \\
$1250^{\circ} \mathrm{C}$ & 92,0 & 86,1 & 86,3 & 70,5 & 59,6 & 55,0 \\
\hline
\end{tabular}




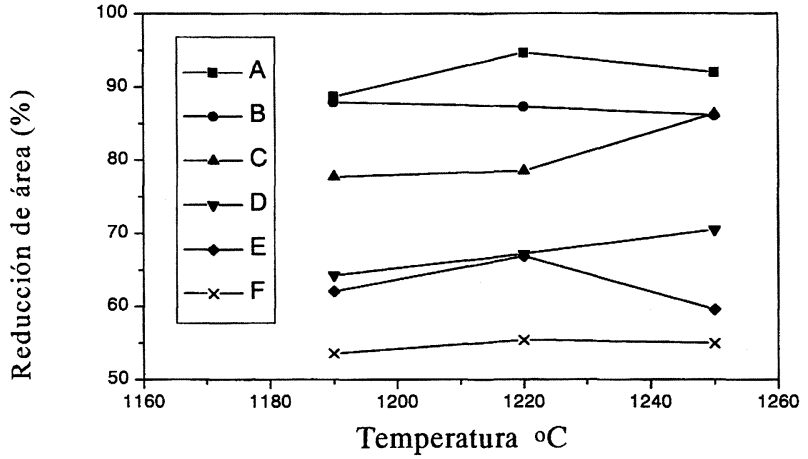

Fig. 1.- Curvas de ductilidad en caliente de los materiales ensayados en el sistema Gleeble 1500 (velocidad inicial de deformación $5 / \mathrm{s}$ ).

FIG. 1.- Hot ductility curves of materials tested on the Gleeble 1500 (strain rate $5 / \mathrm{s}$ ).

grupo $A, B$ y $C$, por una parte, y el $C, D, E$ y $F$ por otra. En el primer caso, se observa una disminución del \% R.A. al aumentar el contenido de azufre. Teniendo en cuenta que la ductilidad del austenítico 316-L es similar a la del 304 cuando los contenidos de azufre son los normales $(0,002 \%)$ (5), pudiéndose obviar, por tanto, el contenido en molibdeno del primero, y que el contenido de inclusiones oxidadas es similar en los tres materiales, la caida de ductilidad desde el nivel de $A$ (referencia) al de $C$ ha de ser atribuida al progresivo aumento del número de inclusiones tipo sulfuro. En los AISI-303, se parte del nivel de ductilidad del material $C$, y se produce un deterioro progresivo en $D, E$ y $F$. En los cuatro materiales, el contenido de azufre es de $\sim 0,3$ $\%$ y el grado de desoxidación disminuye también en el sentido $C, D, E$ y $F$. En este caso, el contenido y la composición de los sulfuros puede considerarse constante, al ser prácticamente iguales los contenidos de azufre y manganeso. La causa de la progresiva menor ductilidad de los materiales $D, E$ y $F$, respecto a ellos mismos y al $C$, es la creciente presencia de inclusiones oxidadas, asociadas a los sulfuros, de forma que aquellas reducen la deformabilidad global de la aleación, debido a su menor ductilidad con respecto a la de la matriz y a la de los propios sulfuros. Esta valoración está soportada experimentalmente por el estudio de secciones longitudinales próximas a las zonas de fractura de las probetas ensayadas. Se ha comprobado cómo los óxidos generan la rotura de la inclusión oxido-sul-

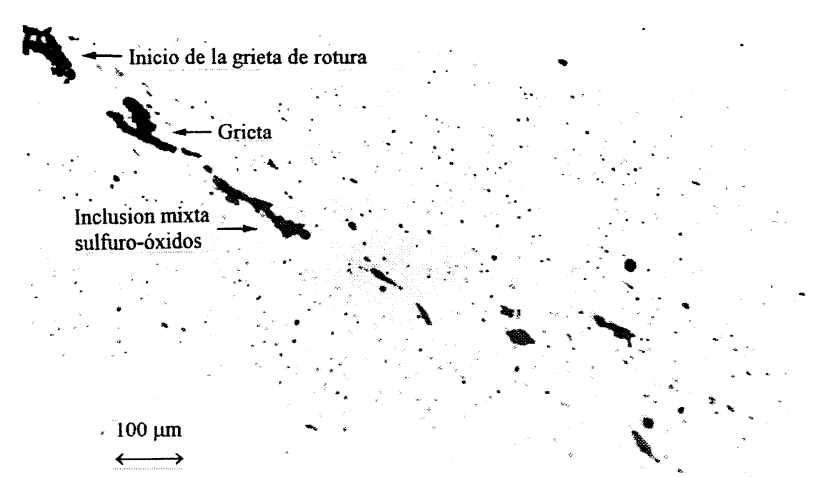

FIG. 2.- Detalle de la generación de grietas por deformación de inclusiones mixtas óxido-sulfuro en la dirección de tracción (sección longitudinal próxima a la zona de fractura, en el material F).

FIG. 2.- Detail of crack formation by the elongation of oxide-sulfide mixed inclusions following tensile direction (lengthwise section close to fracture area, in material $F$ ).

furo, produciéndose el agrietamiento a partir de la interfase entre inclusión y matriz (Fig. 2).

Considerados los factores que podrían intervenir en el resultado de ductilidad del estudio, parece claro que, dada la limitada deformabilidad global del conjunto óxido-sulfuro con relación a los sulfuros simples y a la propia matriz austenítica, la ductilidad de los aceros tipo 303 está directamente relacionada con su índice de desoxidación.

\section{REFERENCIAS}

(1) OHMORI, Y. y MAEhARA, Y. "High-Temperature Ductility of AISI - 310 Steels". Materials Science and Technology. Jun. 1986. Vol. 2 (595-602).

(2) TsugE, S. "Effects of Impurity and Microalloying Elements on Hot Workability of Duplex Stainless Steels". Int. Conf. on Stainless Steels. Chiba 1991 (799-806). ISIJ

(3) Henthornem, M. "Corrosion of Free Machining Stainless Steels". Sulfide Inclusions in Steel, 7-8 Nov.1974. Port Chester - New York (445-458). ASM.

(4) Tegart, W.J. McG. y Gittins, A. "The Role of Sulfides in the Hot Workability of Steels". Sulfide Inclusions in Steel, 7-8 Nov. 1974. Port Chester - NY (199-211). ASM.

(5) Botella, J. ECSC Research project 7210-MA/952, EC 95F3.07a. 